\title{
Locally Activated Mitophagy Contributes to a "built- in" Protection Against Early Burn-wound Progression in Rats Following a Regulation of HIF- 1a
}

\section{Songxue Guo}

The Second Affiliated Hospital Zhejiang University School of Medicine

\section{Quan Fang}

The Second Affiliated Hospital Zhejiang University School of Medicine

\section{Leilei Chen}

First Hospital of Yuhang District Hangzhou

\section{Meirong Yu}

The Second Affiliated Hospital Zhejiang University School of Medicine

\section{Yike Chen}

The Second affiliated hospital Zhejiang University School of Medicine

Nan Li

The Second Affiliated Hospital Zhejiang University School of Medicine

\section{Chunmao Han}

The Second Affiliated Hospital Zhejiang University School of Medicine

Xueqing Hu ( $\nabla$ huxueqing@zju.edu.cn )

The Second Affiliated Hospital Zhejiang University School of Medicine https://orcid.org/0000-00030729-5529

\section{Research}

Keywords: Apoptosis, Burn wound, Hypoxia inducible factor-1凹, Mitophagy, Oxidative stress

Posted Date: August 19th, 2020

DOl: https://doi.org/10.21203/rs.3.rs-55910/v1

License: (9) (1) This work is licensed under a Creative Commons Attribution 4.0 International License. Read Full License 


\section{Abstract}

Background: Deep burn wounds undergo a dynamic progression in the initial or periburn area after insults. The zone of stasis has a higher risk of deterioration and is considered a salvageable target for burn-wound progression. Few studies have explored the role of mitochondrial damage in this process and potential "built-in" self-defense within the human body.

Methods: A classic "comb" scald rat model was established in this study. Histological and blood-flow observation were processed based on hematoxylin-eosin staining and laser analysis. Oxidative and apoptotic status were analyzed by commercial kits. Transmission electron microscope, immunofluorescence staining, and western blot were applied to detect the mitophagy happened in the zone of stasis and potential regulators. Adenovirus-based gene-silence contributed to determine the role of HIF-1 as a regulatory mediator.

Results: We found that burn insults caused typical ischemia and histological deterioration in the zone of stasis, in parallel with increases in oxidative stress and apoptosis. Mitochondrial damage was also involved in the aforementioned changes. Furthermore, we detected typical mitophagy in burn wounds, which was contradictory to the burn-wound conversion. HIF-1区 expression was closely related to the level of mitophagy, while BNIP3 and PARKIN are involved downstream.

Conclusion: We demonstrate that burn-induced mitochondrial impairment contributes to the mobilization of injurious mechanisms in the zone of stasis and that mitophagy provides a more beneficial way to protect against burn-wound progression via the elimination of damaged mitochondria. Our findings offer insights into mitochondrial quality control in burn-wound progression and suggest the novel concept that HIF-1区 may be a potential therapeutic target due to its possible regulatory effects upstream of BNIP3- or PARKIN-mediated mitophagy.

\section{Background}

Deep thermal burns can cause irreversible tissue damage to the integument that can further progress over 3 days[1]. Dynamic conversion, including expansion and deepening, can be observed in the peripheral zones of initial wounds of partial thickness or full thickness burns from $48 \mathrm{~h}$ to $72 \mathrm{~h}$; this is defined as burn wound progression[1-3]. Jackson described three concentric zones (coagulation, stasis and hyperemia from the center to the outer region) to explain the histopathological changes that occur in early burn wounds[4]. The intermediate zone, which undergoes stasis characterized by edema and slow blood flow, progresses to irrecoverable necrosis without appropriate interventions, and the zone of stasis is also considered salvageable and provides an opportunity to prevent cutaneous wound conversion after burn injury[1,2,5]. Efforts to attenuate the early deterioration of initial burn wounds are of great importance.

The burn-induced ischemic status in the zone of stasis is attributed to multiple pathophysiological mechanisms, such as edema, vasoconstriction and hypercoagulability[2,5]. A number of studies have 
suggested that ischemia-induced hypoxia plays a role in changing the membrane permeability of cells or organelles with the help of several cytokines and chemical modulators[6-10]. Ischemia-induced hypoxia is also able to cause a series of secondary biological activities, such as systemic and local inflammation, oxygen free radical burst and increased cell death, all of which are involved in the process of burn wound progression[2,5,11].

Previous studies has reported burn injury causes a series of organ (like skeletal muscle, heart, liver, lung) injury/disorder or cellular damage (like neutrophil) in circulation, attributed to dysfunctional mitochondrial energy metabolism, cellular mitochondrial DNA damage, increased oxidative stress and mitochondrial apoptosis; there is no special attention focus on the detailed role of mitochondria in the progress of early burn wound conversion, although mitochondria are suggested to play a role in mediating burn wound injury[12-17]. Mammalian organisms seem to have developed inner defense systems to protect them from irreversible injuries and also try to determine the negative effects of dysfunctional or damaged cells or organelles[18,19]. Recent studies have suggested that selective autophagy targeting "unhealthy" mitochondria, typically called mitophagy, participates in protecting against ischemia-induced injuries in the brain, kidney and heart, whereas nonselective autophagy is detrimental[$[8,10,20,21]$. The effects of mitophagy are related to mitochondrial quality control, cell survival, and ROS generation[10]. In contrast to the protective benefits of increased mitophagy, mitophagy deficiency promotes hypoxia-induced organ damage. Furthermore, the process of mitophagy involves several regulators, such as BNIP3 and PARKIN, which are thought to be influenced by hypoxia-mediated changes in the intracellular or extracellular environment[19,22].

In our previous study, we observed fluctuating changes in autophagy markers in the zone of stasis at the early stage and a peak at $24 \mathrm{~h}$ postburn[23]. However, it was challenging to determine the role of these changes and the involvement of cellular structure in burn wound progression due to the different types of pathological damage and injury mechanisms[23]. Later, we detected obvious signs of mitophagy in the zone of stasis by transmission electron microscopy (shown below), which was a novel finding. Herein, we hypothesized that mitophagy plays a role in eliminating damaged mitochondria in the zone of stasis and further protects against burn wound progression by relieving oxidative stress and apoptosis. In this study, we aimed to test the abovementioned hypothesis and explore the potential mechanism of regulation involved in this built-in defense system.

\section{Methods}

\section{Experimental animals and the "comb" scald model}

Adult male Sprague-Dawley rats weighing approximately (200-250 g) were purchased from the Shanghai Slac Laboratory Animal Company (Shanghai, China). Adult male Sprague-Dawley rats (weighing approximately 200- $250 \mathrm{~g}$ ) were purchased from the Shanghai Slac Laboratory Animal Company (Shanghai, China). The present study was performed according to protocols approved by the Committee on Animal Care and Use of the Second Affiliated Hospital Zhejiang University School of Medicine (2019- 
331), and strictly followed the National Institutes of Health Guidelines for the Care and Use of Laboratory Animals.

The "comb" scald model was established according to previous reports[23,24]. Specifically, a customized rectangular brass comb (with a transverse area of approximately $20 \mathrm{~mm} \times 10 \mathrm{~mm}$ ) was boiled in $100{ }^{\circ} \mathrm{C}$ water for $5 \mathrm{~min}$ and then applied to the shaved skin surface of the dorsum of rats for $20 \mathrm{~s}$ after anesthesia was induced (sodium pentobarbital, Sigma, St. Louis, MO, USA, $50 \mathrm{mg} / \mathrm{kg}$, intraperitoneal), as shown in Fig. 1a. A row of four bands of full-thickness burns $(20 \mathrm{~mm} \times 10 \mathrm{~mm})$ was made with three interspaces of uninjured skin $(20 \mathrm{~mm} \times 5 \mathrm{~mm})$ to represent the zone of stasis. The burn wound area occupied approximately $4 \%$ of the total body surface area (TBSA). For the sham group, the brass comb was heated in $25^{\circ} \mathrm{C}$ water and applied to the shaved dorsum of rats after anesthesia. During the operation, the breathing and heart rates of the burned rats were carefully monitored to ensure that all of the rats were under anesthesia and pain-free and were then allowed to recover from the anesthesia. Moreover, all rats were housed in individual cages and given $0.25 \mathrm{mg} / \mathrm{kg}$ buprenorphine via subcutaneous injection immediately and every $12 \mathrm{~h}$ after the burn injury for analgesia. In terms of painrelieving intervention, pain and distress scales were used to assess pain in the burned rats immediately and every $6 \mathrm{~h}$ after recovery from anesthesia and informed medical treatment[25].

\section{Research design and sample collection}

Forty-eight animals were randomly assigned to 6 groups: the sham group $(n=8)$ and five burn groups ( $n$ $=8$ per group) at different time points. The five burn groups were euthanized using an overdose of sodium pentobarbital $6,12,24,48$ or $72 \mathrm{~h}$ postburn, whereas the sham group was euthanized $72 \mathrm{~h}$ after pseudo-operation. One band of interspace skin surrounded by $2 \mathrm{~mm}$ of burned tissue on each side (20 $\mathrm{mm} \times 9 \mathrm{~mm}$ ) was harvested from each burned rat and stored in $4 \%$ paraformaldehyde at $4{ }^{\circ} \mathrm{C}$ for subsequent histological and immunofluorescence analyses, and the remaining two bands of interspace skin (without burned tissue; $20 \mathrm{~mm} \times 5 \mathrm{~mm}$ ) from each burned rat were stored at $-80^{\circ} \mathrm{C}$ for Western blot assays. The corresponding bands of unburned skin from each rat in the sham group were also collected as control samples. The general design of this study is shown in Fig. 1b.

\section{Local blood flowgraphy}

A PeriCam PSI system (Perimed, Sweden), which is based on laser speckle contrast analysis (LASCA) technology, was applied to evaluate the local blood flow changes in the burn wounds. Real-time images showing tissue blood perfusion were captured with the help of special PIMSoft software.

\section{Histological preparation and pathologic examination}

Cutaneous samples were biopsied and fixed in $4 \%$ paraformaldehyde, embedded in paraffin and sectioned at a thickness of $3 \mu \mathrm{m}$ using a rotary microtome (RM2245, Leica, Solms, Germany). After deparaffinization and rehydration, hematoxylin and eosin (HE) staining was performed for histological 
examinations, and the tissue slices were observed and imaged under a microscope (DM2500, Leica, Solms, Germany). Five visual fields (200x) from every slice were randomly selected for analysis.

\section{Oxidative stress assessment}

Burn wound tissue homogenates were analyzed with a thiobarbituric acid reactive species (TBARS) assay kit (KGT003-1, KeyGEN, Nanjing, China) to determine the MDA levels, which are expressed as $\mathrm{nmol} / \mathrm{mg}$ protein. Tissue glutathione peroxidase (GPX) and superoxide dismutase (SOD) activities in the zone of stasis were measured separately using commercial assay kits from KeyGEN Biotech (KGT010 and KGT00150, Nanjing, China) according to the manufacturer's recommended protocol. The results are expressed as $\mathrm{nmol} / \mathrm{min} / \mathrm{mg}$ protein and $\mathrm{U} / \mathrm{mg}$ protein. The absorbance values were measured using a microplate reader (Model 680 Microplate Reader, Bio-Rad, CA, USA).

\section{TUNEL staining}

A commercial cell-death detection kit purchased from Roche Diagnostics (Indianapolis, IN, USA) was used for TUNEL staining according to the manufacturer's recommended protocol. The processed slices were observed under a microscope (DM2500, Leica, Solms, Germany), and representative images were captured. The index of apoptosis was determined and is presented as the percentage of apoptotic cells among all the cells counted in a double-blind manner. At least three visual fields per slide and five slides per group were evaluated by two investigators.

\section{Transmission electron microscope-based detection of ultrastructure}

Full-thickness skin tissues from the zone of stasis were dissected from the dorsum of sham-treated and scalded rats for electron microscopy. Briefly, the skin tissues were cut into pieces, immediately fixed in $2.5 \%$ glutaraldehyde in phosphate buffer $(0.1 \mathrm{M}, \mathrm{pH} 7.0)$ (National CHEM, China) at $4{ }^{\circ} \mathrm{C}$ overnight, postfixed in 1\% osmium tetroxide (National CHEM, China) for 1-2 $\mathrm{h}$, and dehydrated in a graded series of ethanol. Then, the specimens were placed in a 1:1 mixture of absolute acetone (National CHEM, China) and the final Spurr resin mixture (SPI-CHEM, West Chester, PA) for $1 \mathrm{~h}$ at room temperature, transferred to a 1:3 mixture of absolute acetone and the final resin mixture for $3 \mathrm{~h}$ and finally moved to a pure final Spurr resin mixture overnight. The prepared specimens were sectioned into 70-nm-thick sections with a microtome (EM UC7, Leica, Germany). The sections were sequentially stained with uranyl acetate (SPICHEM, West Chester, PA) and alkaline lead citrate (National CHEM, China) for 5 to 10 min, respectively. The stained tissue sections were examined initially at low magnification $(1,5000 x)$ to identify representative structures. The cells in the selected zone were then examined at high magnification $(3,0000 \times$ or $50,000 \times)$ to reveal mitochondria. Briefly, digital images with scale bars were collected by TEM. Representative digital images with scale bars, which showed cellular mitochondria undergoing the process of mitophagy in the zone of stasis, were captured by transmission electron microscopy (H7650, Hitachi, Japan).

\section{Immunofluorescence staining}


Prepared slices were washed in PBS for $10 \mathrm{~min}$ and then boiled in $0.01 \mathrm{mmol}$ citrate buffer $(\mathrm{pH}=6)$ for $10 \mathrm{~min}$ for antigen retrieval. After incubation with hydrogen peroxide for $10 \mathrm{~min}, 5 \%$ bovine serum albumin (BSA) was applied as the blocking solution for $20 \mathrm{~min}$ at room temperature. Without being washed, the sections were incubated with an anti-CC3 (1:200; \#9664, Cell Signaling Technology, MA, USA), anti-p-Bad (1:200; sc-12969-R, Santa Cruz, CA, USA), anti-LC3 II (1:200; \#4060, Cell Signaling Technology, MA, USA) or anti-TOMM20 (1:200; sc-12969-R, Santa Cruz, CA, USA) antibody overnight at 4 ${ }^{\circ} \mathrm{C}$. After being rinsed with PBS, the sections were incubated with Alexa 488 (1:50; Abcam, China)- or Cy3 (1:50; BA1032, Boster, Wuhan, China)-labeled goat anti-rabbit secondary antibodies for $2 \mathrm{~h}$ at $37^{\circ} \mathrm{C}$ in the dark. The sections were rinsed and then mounted with Vectashield mounting medium with DAPI (Vector Laboratories, Burlingame, CA, USA). All slices were observed and photographed under a fluorescence microscope (DM5500B, Leica, Solms, Germany).

\section{Quantitative real-time PCR (qRT-PCR) detection}

The expression level of HIF-1区 in the zone of stasis was analysed via qRT-PCR. Briefly, total RNA was isolated from tissues with TRIzol Reagent (Invitrogen, Carlsbad, CA, USA) and RNase-Free DNase I (Qiagen, Duesseldorf, Germany). The SuperScript First- Strand Synthesis System for reverse transcription PCR (Invitrogen, Carlsbad, CA, USA) was applied to synthesise cDNAs, and RNA and CDNA concentrations and purities were measured via BIO-RAD spectrophotometry (SmartSpecTM Plus, BIO-RAD, CA, USA). The primers were designed using Primer Premier 6.0 software, as a forward primer 5'-

CGTGCCCCTACTATGTCGCTTT-3' and a reverse primer 5'-GTCTTCTGCTCCATTCCATCCTGT-3' and were synthesised by Shanghai Biological Engineering Co., Ltd. (Shanghai, China). PCR amplifications were conducted using the Power SYBR® Master Mix (Invitrogen, Carlsbad, CA, USA) in an iQTM 5 Real-time PCR system (BIO-RAD, CA, USA). Expression levels were assessed relative to that of GAPDH, as an internal standard, and the primer detail is shown as forward 5'-GAAGGTCGGTGTGAACGGATTTG-3' and reverse 5'-CATGTAGACCATGTAGTTGAGG TCA-3'. Relative quantification of the target gene expression level was conducted using the $2^{-\Delta \Delta C t}$ method.

\section{Western blot analysis}

Skin samples were collected, frozen in an ultra-low freezer and cut into pieces, which were lysed with RIPA lysis buffer (AR0105, Boster, Wuhan, China) for $1 \mathrm{~h}$ on ice. The lysates were mixed with loading buffer and centrifuged at $14,000 \mathrm{xg}$ for $10 \mathrm{~min}$, and the protein samples were subjected to SDS-PAGE and then transferred onto nitrocellulose membranes via electrophoresis. In addition, aliquots of the samples were used to determine the protein concentration of each sample using a bicinchoninic acid (BCA) kit (KGPBCA, KeyGEN, Nanjing, China). Subsequently, the membranes were incubated in blocking buffer for 2 $\mathrm{h}$ and incubated overnight at $4{ }^{\circ} \mathrm{C}$ with the following primary antibodies: anti-cleaved caspase 3 (1:1000; \#9664, Cell Signaling Technology, MA, USA), anti-cleaved caspase 9 (1:1000; \#9507, Cell Signaling Technology, MA, USA), anti-p-Bad (1:2000; ab129192, Abcam, Cambridge, UK), anti-Bad (1:2000; ab62465, Abcam, Cambridge, UK), anti-Cytochrome C (1:1000, ab13575, Abcam, Cambridge, UK), anti-TOMM20 (1:500; ab56783, Abcam, Cambridge, UK), anti-SQSTM1 (1:200; ab56416, Abcam, Cambridge, UK ), anti- 
LC3II (1:1000; \#4108, Cell Signaling Technology, MA, USA), anti-PARKIN (1:1000; ab179812, Abcam, Cambridge, UK), anti-BNIP3 (1:1000; ab109362, Abcam, Cambridge, UK), anti-BNIP3L (1:1000; ab109414, Abcam, Cambridge, UK), anti-FUNDC1 (1:200; ab173226, Abcam, Cambridge, UK), and anti-HIF-1 (1:10000; ab179483, Abcam, Cambridge, UK). GAPDH (1:10000; ab181602, Abcam, Cambridge, UK) was used as a control on the same membranes. After the application of secondary antibodies, the bands were detected with West Dura Extended Duration Substrate (Pierce, USA), and exposed X-ray films (Kodak, USA) were analyzed using Bandscan 5.0 software based on comparisons with GAPDH bands.

\section{Adenovirus delivery}

Under sodium pentobarbital anesthesia (50 mg/kg, intraperitoneal), mCherry-labeled adenoviruses (Advs, designed, produced and identified by Bioomin Technology Corp., Ltd., Shanghai, China), including Adnegative control (NC) and Ad-shHIF-1区, were injected subcutaneously into selected locations on the shaved dorsum of rats (nine locations) (Fig. 6A). Subsequent experiments were performed a minimum of $72 \mathrm{~h}$ after infection to allow sufficient time for gene silencing, and the effects of the adenoviruses were evaluated by a Bio-Real in vivo imaging system (IVIS spectrum, Perkin Elmer, MA, USA).

\section{Statistical analysis}

The data are presented as the means \pm standard deviations (SDs). GraphPad Prism version 7 (San Diego, CA, USA) was used for the statistical analyses. Multiple comparisons were performed via one-way analysis of variance (ANOVA) followed by Bonferroni post hoc tests. Unpaired t-tests were used for comparisons between two groups. $P$-values less than 0.05 were considered statistically significant.

\section{Results}

\section{Burn insult causes typical hemodynamic and histopathological changes in the zone of stasis and related zones of burn wounds with time.}

After burn, the space between two coagulation zones showed a tendency to deteriorate and merge over time (Fig. 2a, upper row). Simultaneous laser detection showed typical reduction of blood flow in the zones closest to the direct thermal injury after insult, while the all three interval zones ( $R O I 2,3,4)$, representing the zones of stasis, exhibited low blood flow $6 \mathrm{~h}$ and $12 \mathrm{~h}$ postburn ( $6 \mathrm{~h}$ ROI 2: 161ه $68 \mathrm{PU}$, ROI 3: $188 \rrbracket 70$ PU, ROI 4: $109 \rrbracket 52$ PU; 12 h ROI 2: $164 \rrbracket 61$ PU, ROI 3: $184 \llbracket 70$ PU, ROI 4: $95 \rrbracket 45$ PU ), compared to the perfusion of the group post burn immediately (ROI 2: $222 \otimes 87 \mathrm{PU}, \mathrm{ROI} 3: 217 \otimes 54 \mathrm{PU}$, $\mathrm{ROI} 4: 91 \llbracket 43 \mathrm{PU}$ ), and then gradually improved stasis from $24 \mathrm{~h}$ to $72 \mathrm{~h}$ postburn (ROI 2: 232-305 $₫ 89-98$ $\mathrm{PU}, \mathrm{ROI} 3 \mathrm{~s}: 260-295 \otimes 63-96 \mathrm{PU}, \mathrm{ROI} 4: 129-200 \otimes 48-67 \mathrm{PU})$; the uninjuried region showed a relatively stable perfusion with a value of around $140 \mathrm{PU}$ in all time (Fig. 2a lower row and 2b). In terms of pathological changes, hematoxylin and eosin (HE) staining was used to observe representative characteristics of burn wounds, such as thinning of the epidermis, epithelial nuclei elongation, swelling of the dermis with alterations in collagen, and inflammatory cell infiltration, and all of the abovementioned changes became more obvious over time after burn (Fig. 2c). 


\section{Burn injury induces a time-dependent increase in oxidative stress and apoptosis in the zone of stasis}

A lipid peroxidation indicator (MDA) and endogenous antioxidant enzymes (glutathione peroxidase (GPx) and superoxide dismutase (SOD)) were detected to assess oxidative stress in the zone of stasis. Specifically, $6 \mathrm{~h}$ postburn, the MDA level showed a significant increase, while similar changes were also observed in the $12 \mathrm{~h}$ and $24 \mathrm{~h}$ postburn groups (Fig. 3a). The elevated MDA level was obviously decreased in the $48 \mathrm{~h}$ postburn group compared to the other groups except the $72 \mathrm{~h}$ postburn group (Fig. 3a). The MDA level increased slightly $72 \mathrm{~h}$ postburn, but there was no significant difference between the $48 \mathrm{~h}$ and $72 \mathrm{~h}$ postburn group (Fig. 3a). In terms of GPx, all burn groups showed significant differences compared to the sham group, and the activity of GPx decreased gradually after burn injury (Fig. 3a). Although SOD activity in the burn wound displayed no obvious changes $6 \mathrm{~h}$ postburn, significant decreases were observed from $12 \mathrm{~h}$ to $72 \mathrm{~h}$ after burn (Fig. 3a). The lowest activity of SOD was detected $48 \mathrm{~h}$ postburn (Fig. 3a).

TUNEL staining was performed, and apoptotic cells in the stasis zone, which were visualized as brownlabeled nuclei, were observed in all burn groups. Apoptotic cells were subsequently counter to determine the apoptosis index (representing the percentage (\%) of apoptotic cells), which increased obviously from

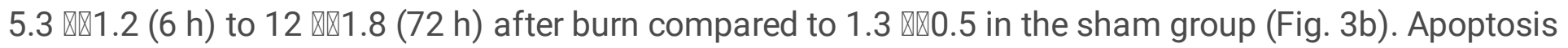
peaked $48 \mathrm{~h}$ and $72 \mathrm{~h}$ postburn (Fig. 3b).

The caspase cascade contributes to the stress-induced mitochondrial response and cleaves downstream cellular targets, ultimately inducing apoptosis. The activating caspase Caspase 9 and the effector caspase Caspase 3 play important roles in the mitochondrial apoptotic pathway. Immunofluorescence staining revealed that burn insult induced a gradual increase in the distribution of cleaved caspase 3 (CC3, active status), which presented as lime green-labeled structures, in the wound over time (Fig. 3c, left). A similar tendency was also found by immunoblotting for CC3.The significant upregulation was observed since $6 \mathrm{~h}$ postburn and continued until $72 \mathrm{~h}$ postburn compared to that in the sham group (Fig. $3 \mathrm{c}$, right). The expression levels in the $12 \mathrm{~h}$ and $24 \mathrm{~h}$ postburn groups were not different, while peak expression was found in both the $48 \mathrm{~h}$ and $72 \mathrm{~h}$ postburn groups. The expression of upstream cleaved caspase 9 (CC9), like that of CC3, was observed to increase at different time-points after burn injury (Fig. $3 \mathrm{c}$ right). The expression of CC9 was significantly upregulated from $12 \mathrm{~h}$ postburn until $72 \mathrm{~h}$ postburn (Fig. 3c), while only a slight increase appeared in the $6 \mathrm{~h}$ postburn group. CC9 expression increased more than twice as much in the $12 \mathrm{~h}$ and $24 \mathrm{~h}$ burn groups as in the sham group, while CC9 expression in both the $48 \mathrm{~h}$ and $72 \mathrm{~h}$ burn groups increased by more than threefold (Fig. $3 \mathrm{c} \mathrm{right).}$

\section{Burn insult induces mitochondrial damage and the activation of the mitochondria-related apoptosis pathway in the zone of stasis}

In response to external stress, mitochondrial stress pathways are aroused, following membrane potential change and even structural instability. After burn, the distribution of violet-labeled phosphorylated Badpositive cells (merged) increased obviously in the zone of stasis with time (Fig. 4a). Immunoblotting also verified the gradual increase in the phosphorylation of Bad at different times after burn, and the peak 
could be detected both $48 \mathrm{~h}$ and $72 \mathrm{~h}$ postburn (Fig. $4 \mathrm{~b}$ ). The release of cytochrome $\mathrm{C}$ (Cyto $\mathrm{C}$ ) was detected based on the level of cytosolic Cyto $\mathrm{C}$, which suggested an augmentation of Cyto $\mathrm{C}$ beginning 6 $\mathrm{h}$ postburn (Fig. $4 \mathrm{~b}$ ). The level of Cyto $\mathrm{C}$ in the cutaneous cytoplasm increased threefold $12 \mathrm{~h}$ postburn, nearly fourfold $24 \mathrm{~h}$ and sixfold $48 \mathrm{~h}$ postburn compared to that in the sham group, while it peaked $72 \mathrm{~h}$ after burn injury (Fig. 4b). Further detection of mitochondrial(mt) DNA, measured as the mt-Atp6:Rpl13 ratio, an indicator of the relative amounts of mitochondria, decreased significantly beginning $6 \mathrm{~h}$ postburn (Fig. 4c). The lowest mt-Atp6:Rpl13 ratio was found $12 \mathrm{~h}$ after burn, and then the decrease reversed gradually from $24 \mathrm{~h}$ to $72 \mathrm{~h}$; at these time points, the ratios were still remarkably lower than that of the sham group (Fig. 4c). Combined with the aforementioned burn-induced apoptosis, these changes related to Bad phosphorylation, Cyto $\mathrm{C}$ release and mt-DNA provided a clue regarding mitochondrial damage. The subsequent mobilization of the mitochondria-related apoptotic pathway was also considered meaningful for the regulation of apoptosis in the stasis zone.

\section{Mitophagy is detected in burn wounds in the early stage after injury and shows a fluctuation with time}

Typical mitophagy was detected by transmission electron microscope (TEM) beginning $6 \mathrm{~h}$ after burning in the stasis zone; deformed and damaged mitochondria encircled by forming autophagosomes were observed (Fig. 5a). We observed the coexpression of LC3 II with the outer mitochondrial membrane marker TOMM 20 by immunofluorescence detection, which was used as an indicator of selective mitophagy in the wound tissue (Fig. 5b). Moreover, immunoblot analysis revealed a downregulation of TOMM20 expression with time, although a significant increase was detected in the $72 \mathrm{~h}$ postburn group compared to the $48 \mathrm{~h}$ postburn group. SQSTM1, as a bridge between polyubiquitinated cargo and autophagosomes, usually interacts with an autophagy modifier of the microtubule-associated protein 1 light chain 3 (MAP1 LC3/LC3) family. In this study, we detected that SQSTM1 protein expression showed a downregulation within $12 \mathrm{~h}$ postburn, but a gradual increase with time appeared since $24 \mathrm{~h}$ and peaked $48 \mathrm{~h}$ and $72 \mathrm{~h}$ after burn. Meanwhile, the ratio of LC3 II to LC3 I increased significantly from $6 \mathrm{~h}$ to $24 \mathrm{~h}$ in the zone of stasis and then decreased beginning $48 \mathrm{~h}$ postburn. The $48 \mathrm{~h}$ postburn groups and the sham group showed similar LC3 II ratios, while the ratio of the $72 \mathrm{~h}$ group was obviously downregulated compared to those of other groups. The ratio of LC3 II to LC3 I peaked at $6 \mathrm{~h}$ postburn, and there were significant decreases $12 \mathrm{~h}$ and $24 \mathrm{~h}$ after burn; however, no difference was found between the $12 \mathrm{~h}$ and $24 \mathrm{~h}$ postburn groups (Fig. $5 \mathrm{c}$ ). All of the above results indicated that mitophagy occurred beginning $6 \mathrm{~h}$ postburn and that it was more obvious in the earlier period after burn (6 to $24 \mathrm{~h}$ ).

\section{Both BNIP3 and PARKIN are involved in the regulation of mitophagy in burn wounds, while HIF-1区 acts upstream}

To explore potential participants in the regulation of mitophagy in the zone of stasis, we detected previously reported regulators, such as PINK1/PARKIN, BNIP3, BNIP3L, and FUNDC1, by immunoblotting (Fig. 6a). PARKIN showed a significant upregulation beginning $6 \mathrm{~h}$ postburn and increased gradually until $24 \mathrm{~h}$, and then its expression showed a decrease since $48 \mathrm{~h}$ postburn. We also observed a remarkable increase in PINK1 and BNIP3 expression, which presented a gradual decrease after peaking $6 \mathrm{~h}$ postburn. 
A significant upregulation of BNIP3L was found in all groups expect the $24 \mathrm{~h}$ group, and the lowest levels of expression were observed in the $72 \mathrm{~h}$ postburn group, which exhibited significantly different levels compared to the other four groups. Although the protein level of FUNDC1 in the zone of stasis was obviously increased at all selected time points after burn, there was a significant difference between the levels of the $48 \mathrm{~h}$ group and the other four groups.

Further investigation by real-time PCR and western blotting showed that both the transcription and expression of HIF-1区 increased significantly beginning $6 \mathrm{~h}$ after burn and peaked at 12 or $24 \mathrm{~h}$, following the various ischemic/hypoxic conditions in burn wounds (Fig. 6B). Subsequently, a slight change in HIF-

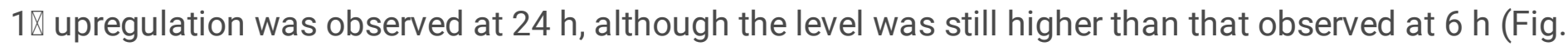
6b). Nevertheless, the levels of HIF-1 1 at $48 \mathrm{~h}$ and $72 \mathrm{~h}$ were obviously lower than those at $12 \mathrm{~h}$ or $24 \mathrm{~h}$ postburn, which were similar to those at $6 \mathrm{~h}$ but still higher than those in the sham group (Fig. 6b).

Compared with mitophagy markers, BNIP3 and PINK1 seemed to be related to the regulation of mitophagy due to their autologous protein expression. The reported mediating role of HIF-1区 assisted in establishing a link between HIF-1】 and BNIP3, which was also able to influence PARKIN through PINK1. However, the delayed peak in the expression of PARKIN indicated another role independent of BNIP3/PINK1, suggesting a closer relationship with HIF-1区.

\section{HIF-1区 silencing impairs BNIP3 and PARKIN-mediated mitophagy and aggravates mitochondria-related apoptosis in the zone of stasis}

Fluorescence-labeled adenoviruses (Advs) were used to inhibit the regulation of HIF-1】 in burn wounds. In vivo imaging indicated the effectiveness of local mCherry-labeled Adv transfection in the cutaneous tissue of rats (Fig. 7a). Immunoblotting further demonstrated the deficiency in HIF-1Q in the zone of stasis of Adv-HIF-1ه shRNA-injected rats, while Adv-negative control (NC) shRNA showed no effect on the

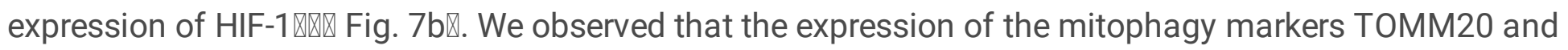
SQSTM1 increased significantly after the administration of Adv-HIF-1】 shRNA, while the ratio of LC3II to LC3I decreased remarkably (Fig. 7c). Ad-NC shRNA showed no or a relatively slight effect on the expression of TOMM20, SQSTM1 and LC3II. On the other hand, in the burn groups, $24 \mathrm{~h}$ after burn, the downregulation of TOMM20 and SQSTM1 was reversed by Adv-HIF-1囚 shRNA, while LC3II ratio showed a dramatic decrease (Fig. 7c). Furthermore, the investigation of mitochondria-related apoptosis showed that HIF-1区 silencing helped to increase Cyto $\mathrm{C}$ release in both the sham and burn groups and CC3 expression in the sham group, suggesting increased apoptosis in the zone of stasis (Fig. 7d). However, Adv-NC shRNA downregulated Cyto $\mathrm{C}$ release and CC3 expression significantly in the burn groups, whereas it increased Cyto $C$ release significantly and showed no effect on CC3 expression in the sham groups (Fig. 7d). Finally, we investigated the potential effect of HIF-1区 on the mitophagy regulators BNIP3 and PARKIN. Protein blotting revealed that silencing induced by Adv-based HIF-10silence resulted in significant downregulation of BNIP3 and PARKIN in both the sham and burn groups, while Adv-NC shRNA only decreased the expression of BNIP3 and PARKIN in the burn groups (Fig. 7e). 


\section{Discussion}

Throughout the past decades, multiple efforts have been made to attenuate and prevent thermal injury and secondary local or systemic injuries after burn; however, ideal prognosis has not accompanied the huge economic costs. Emergency treatment for early burn wound progression provides the opportunity to relive the detrimental effects of traumatic thermal injury and improve outcomes[2,26]. Current knowledge suggests that the ischemic/hypoxic condition of stasis, caused by hypoperfusion, microthrombus formation or blood stasis, contributes to a series of downstream activities, such as ROS burst, cell death, and inflammation[2]. Although impaired mitochondria are regarded as an important source of excessive ROS and regulators of apoptosis in several ischemic organs and although mitophagy is a classical process that eliminates the effects of mitochondria, the role of mitochondrial in the development and protection of burn wounds is still unclear[9,19]. In the present study, we first investigated the potential protective effect of mitophagy against burn wound progression as a built-in defense mechanism. The results indicated the following: 1) mitochondrial damage occurs in the zone of stasis over the first 72 hours following ischemic/hypoxic injury postburn, which is accompanied by a gradually progressive deterioration of the burn wound; 2 ) impaired mitochondria contribute to decreased oxidative phosphorylation and disability of energy production, resulting in increased oxidative stress in the zone of stasis, as well as activated mitochondria-related apoptosis; and 3) mitophagy occurs within the first 3 days after burn and is great potential to stabilize mitochondrial to reduce burn wound conversion by targeting damaged mitochondria, while HIF-1囚 and BNIP3 and PARKIN seems to work as an upstream regulatory pathway. Hence, the present findings provide further evidence that confirm our notion that mitophagy protects against burn wound progression and shed light on HIF-1区 as a potential target for burn wound therapy.

Except for during direct thermal insult, secondary pathophysiologic processes begin in the early stage after burn[1,27-32]. Ischemia and low-flow status can be easily observed in the local organs or systemic circulation of burned patients and animal models, suggesting the existence of hypoxia[5,30,32,33]. In burn wounds, we observed obvious ischemia/hypoperfusion in the stasis zone by laser-speckle detection; these observations corresponded to typical pathological changes in cutaneous tissue and were consistent with previous reports[34,35]. Interestingly, we found that extreme ischemia did not initially accompany the severest tissue injury in the intermediate zones, whereas further deterioration seemed to occur when blood flow slightly improved (but hypoperfusion state was still present) at a later point (48 or $72 \mathrm{~h}$ ). All these results indicate that there is an intermediate in the communication between blood flow/oxygen supply and the machineries of burn injury. As crucial organelles that sense oxygen in mammalian cells, mitochondria are the most sensitive of ischemic/hypoxic injury[9,36]. In ischemic/hypoxic injury, an insufficient oxygen supply causes mitochondrial swelling, the loss of the mitochondrial membrane potential and disorders in ATP production, which lead to the perturbation of mitochondrial homeostasis and quality $[9,10]$. Moreover, impaired mitochondria ultimately lead to excessive ROS generation and the activation of mitochondria-mediated apoptosis following the dysregulation of the electron transport chain and molecular pathways $[9,10]$. Our previous studies demonstrated that antioxidants can attenuate burn wound progression by decreasing oxidative stress 
and activating the mitochondria-mediated apoptotic pathway[37,38]. In the present study, we investigated time-dependent changes in oxidative stress and apoptosis in the zone of stasis over a period of $72 \mathrm{~h}$ postburn. Ischemic/hypoxic injury-induced ROS production by mitochondria has been reported to overwhelm the inner antioxidant capacities of cells and eventually cause mitochondrial lipid peroxidation, the disruption of calcium ion homeostasis and even cellular DNA damage[9]. The difference was that MDA peaked within $24 \mathrm{~h}$ postburn and that the lowest levels of antioxidative enzyme activities were observed in the late stage, which indicated that mitochondrial ROS may play a role besides oxidative damage in burn wound progression. On the other hand, we also found further evidence to support the existence of mitochondrial damage and related apoptosis. The gradual increase in caspase activation in the zone of stasis was accompanied by increased phosphorylation of Bad (an important member of the Bcl-2 family) and release of Cyto $\mathrm{C}$, both of which strongly suggested the role of mitochondria in the control of burn-induced apoptosis and the decreased ratio of mtDNA.

To our knowledge, organisms on earth have developed meticulous built-in mechanisms to perceive environmental changes and respond to diverse insults. Recently, selective autophagy, including aggrephagy, mitophagy and cilia, has aroused researchers' interests because of its ability to degrade specific cellular targets and its potential to maximize clinical benefits[39]. Ischemia/hypoxia have been reported to selectively activate mitophagy to decrease the abnormal accumulation of impaired mitochondria for both mitochondrial quantity and quality control[19,22]. Zhang et al. described the protective effect of ischemia/hypoxia-induced mitophagy against worsening I/R heart injury through the regulation of mitochondrial quality/quantity and platelet activation[21]. Tang et al. suggested the beneficial effect of PINK1/PARKIN and BNIP3-mediated mitophagy in protecting kidneys from I/R injury by attenuating tubular apoptosis, inflammatory infiltration, and increased ROS production[8,40]. Furthermore, mitophagy is thought to mediate the protective effect of ischemic preconditioning in kidneys by targeting a series of biological events, including damaged mitochondrial clearance, mitophagosome formation, and mitochondrial depolarization[41]. In addition, crucial roles for mitophagy in affording neuroprotection against IR cerebral injury and alleviating metabolic dysfunction have also been reported $[20,42-44]$. Under tumor-related hypoxic conditions, the activation of mitophagy is involved in assisting tumor cell survival and promoting cancer, while BNIP3- or BNIP3L-mediated mitophagy may act as a suppressor of tumorigenesis and metastasis in breast cancer or xenograft conditions[45-47]. In the present study, the detection of TOMM20 and SQSTM1 in the outer mitochondrial membrane provided a way to assess the level of mitophagy with the general autophagy marker LC3 II. We found dramatically increased mitophagy accompanied by a lower severity of burn injury and slightly increased apoptosis in the zone of stasis within the first $24 \mathrm{~h}$ postburn, whereas weakened mitophagy activation was observed with more severe tissue damage and remarkable activation of apoptosis. Moreover, ROS level was constantly significantly increased within $72 \mathrm{~h}$ postburn; however, a higher level of oxidative stress in the burn wound within $24 \mathrm{~h}$ postburn seemed to be linked with greater activation of mitophagy. Ischemia/hypoxia-induced excessive accumulation of ROS can promote cellular autophagy via some regulatory pathways following increased oxidative stress and mitochondrial dysfunction under diverse pathological conditions[22,47]. In turn, selective autophagy, especially mitophagy, is able to lower 
oxidative stress by eliminating damaged mitochondria and decreasing secondary ROS release[19]. The abovementioned interaction seems to be an appropriate explanation for the analogous tendencies of oxidative stress and mitophagy, which further indicates the dual effects of ROS in burn wound progression in inducing molecular signals and causing direct damage.

Oxygen sensation and adaption are essential for multiple physiological processes and diseases[48]. Previous researchers, who were awarded the Nobel Prize in 2019, explored and described the detailed molecular machinery involved in the process of oxygen availability on the levels of oxygen[48-52]. As a crucial player, HIF-1】 exhibits significant regulatory effects under hypoxic conditions in various diseases or ischemic injuries[53]. Specifically, a low oxygen level enhances HIF-1ه stabilization via oxygendependent hydroxylation[54]. Xing et al. reported HIF-1囚 upregulation in the healing region of burn wounds (similar to the location of the stasis zone) beginning $48 \mathrm{~h}$ after burn and peaking on day 3 after burn, as is observed under hypoxic conditions[55]. These findings support the importance of HIF-1区 in promoting angiogenesis and vasculogenesis in burn wound repair[55]. In response to hypoxia, mitophagy mediated by both receptors (BNIP3, BNIP3L, and FUNDC1) and kinases (PINK/PARKIN) can be activated via both transcriptional and posttranslational modifications, while existing evidence suggests that BNIP3 and BNIP3L are linked to the regulation of HIF-1 [22]. Presently, we investigated the time-related expression of potential regulators on mitophagy in the zone of stasis and found that the expression of BNIP3, which is remarkably increased from $6 \mathrm{~h}$ to $24 \mathrm{~h}$ and restored partly from $24 \mathrm{~h}$ to $72 \mathrm{~h}$, corresponds to changes in mitophagy within $72 \mathrm{~h}$ after injury; this suggests a valuable regulatory role of BNIP3 in mitophagy in burn wounds. Based on the Drosophila model, Zhang et al. suggested that hypoxia-induced BNIP3 upregulation can protect PINK1 from proteolytic cleavage and promote PINK1/PARKIN-mediated mitophagy, which indicates upstream regulation of PINK1 and even PARKIN by BNIP3[56]. In the present study, we observed an autologous change in BNIP3 and PINK1 expression after burn, which indicates that PINK1/PARKIN may be induced by BNIP3 to influence mitophagy. The fluctuation in HIF-1区 levels (first increased and then decreased) provides further evidence of the role of a HIF-1ه/BNIP3/PINK1/PARKIN cascade in the regulation of mitophagy; however, the changes in BNIP3 and PINK1 were not completely consistent with the changes in the expression of PARKIN, and the delayed peak in PARKIN was more consistent with a downstream target of HIF-1】. Some researchers have suggested a role for PARKIN in cell division independent of PINK1 [57]. In our recent work, we also found some clues regarding the epigenetic regulation of HIF-1囚 on PARKIN (unpublished). Therefore, we are not able to completely exclude the potential effect of PARKIN on mitophagy independent of BNIP3 and PINK1. On the other hand, BNIP3L and FUNDC1 showed significant upregulation at all time points, but these tendencies did not explain the higher incidence of mitophagy at the earlier stage (within $24 \mathrm{~h}$ ) after burn. To demonstrate the possible regulatory link between HIF-1区 and BNIP3 or PARKIN, we applied adenovirus-based gene silencing to eliminate the effect of burn-induced HIF-1区 upregulation in the zone of stasis $24 \mathrm{~h}$ postburn. The selected time point $(24 \mathrm{~h})$ showed typical increased mitophagy prior to a secondary decrease accompanied by a relatively lower level of apoptosis, which was considered an ideal opportunity for protective interference. The results verified that silencing HIF-1ه led to a decrease in local mitophagy in the sham and burn groups and increased activation of the downstream mitochondrial apoptotic pathway, 
while both BNIP3 and PARKIN were significantly downregulated after ShHIF-1区 administration. These findings suggest the important role of HIF-1】 in regulating tissue mitophagy in early burn wound progression through the involvement of some downstream mediators, such as BNIP3 and PARKIN.

\section{Conclusion}

In summary, the present study provides valuable clues to confirm that mitophagy has great potential to prevent early burn wound progression by eliminating impaired mitochondria and its secondary detrimental effects, such as oxidative damage and apoptosis, in the zone of stasis. As a part of the selfdefense system in mammals, enhanced mitophagy is suggested as a novel strategy for early burn wound therapy, while HIF-1\ serves as a crucial liaison between local hypoxia and mitophagy through downstream BNIP3 and PARKIN. Although a network of mitophagy in burn wound progression is suggested by our study, as shown in Fig. 8, further investigations are still needed to clarify the detailed mechanism by which HIF-1囚 regulated certain mitophagy regulators in burn wounds, as well as the potential effect of mitophagy on tissue inflammation.

\section{Abbreviations}


HIF-10 hypoxia inducible factor-10

\begin{tabular}{|c|c|}
\hline GAPDH囚 & glyceraldehyde-3-phosphate dehydrogenase \\
\hline mt-Atp6 & mitochondrially encoded ATP synthase 6 \\
\hline Rpl13 & ribosomal protein L13囚 \\
\hline mtDNA & mitochondrial DNA \\
\hline TOMM20 & translocase of outer mitochondrial mem- brane 20 homolog (yeast) \\
\hline SQSTM1 & sequestosome 1 \\
\hline BNIP3L & BCL2/adenovirus E1B interacting protein 3-like \\
\hline Adv & adenovirus $\rrbracket$ \\
\hline DAPI & 4',6-Diamidino-2-phenylindole dihydrochloride \\
\hline PINK1 & PTEN induced putative kinase 1 \\
\hline ROS & reactive oxygen species \\
\hline BNIP3 & BCL2 interacting protein 3 \\
\hline TUNEL & terminal deoxynucleotidyl transferase-mediated dUTP nick end labeling \\
\hline ShRNA & short hairpin RNA \\
\hline MDA & malondialdehyde \\
\hline GPx & glutathione peroxidase \\
\hline SOD & superoxide dismutase \\
\hline $\mathrm{CC} 3$ & cleaved caspase 3 \\
\hline CC9 & cleaved caspase 9 \\
\hline Cyto C & cytochrome c \\
\hline TEM & transmission electron microscope \\
\hline MAP1 LC3/LC3 & microtubule-associated protein 1 light chain $3 \rrbracket$ \\
\hline FUNDC1 & FUN14 domain containing 1 \\
\hline NC & negative control \\
\hline Bad & Bcl-2-associated death promoter \\
\hline p-Bad & phosphorylated Bcl-2-associated death promoter \\
\hline $\mathrm{Bcl}-2$ & B-cell lymphoma-2 \\
\hline
\end{tabular}




\section{Declarations}

\section{Ethics approval and consent to participate}

The present study was performed according to protocols approved by the Committee on Animal Care and Use of the Second Affiliated Hospital Zhejiang University School of Medicine (2019-331), and strictly followed the National Institutes of Health Guidelines for the Care and Use of Laboratory Animals.

\section{Consent for publication}

Not applicable.

\section{Availability of data and materials}

The datasets used and/or analyzed during the present study are available from the corresponding author on reasonable request.

\section{Competing interests}

The authors declare no competing financial interests.

\section{Funding}

This work was supported by the following grants: National Natural Science Foundation of China (NSFC) Grants 81671909, 81901958 and Zhejiang Provincial Natural Science Foundation of China Grants: LY18H150004, LY19H150004, LY20H150010.

\section{Authors' contributions}

C.H. and X.H guided the experimental procedures. S.G, L.C. and Y.C. participated in making the experimental animal model. S.G., Q.F., M.Y. and N.L. performed histo/immunostaining, kits detection, qRTPCR and western blotting. S.G., Q.F., C.H. and X.H. participated in the design of the study and the writing of the manuscript. All authors read and approved the final manuscript.

\section{Acknowledgements}

We are especially grateful to Prof. Dennis P. Orgill from Brigham and Women's Hospital Harvard medical School for reviewing our manuscript and providing some advices to improve it. We also appreciate the assistance from Bio-ultrastructure analysis Lab. of Analysis center of Agrobiology and environmental sciences, Zhejiang University for TEM detection.

\section{References}

1. Singh V, Devgan L, Bhat S, Milner SM. The pathogenesis of burn wound conversion. Annals of Plastic Surgery. 2007;59:109-15. 
2. Salibian AA, Rosario ATD, Severo LDAM, Nguyen L, Banyard DA, Toranto JD, et al. Current concepts on burn wound conversion-A review of recent advances in understanding the secondary progressions of burns. Burns. 2016;42:1025-35.

3. Orgill DP. Excision and skin grafting of thermal burns. N. Engl. J. Med. 2009;360:893-901.

4. JACKSON DM. [The diagnosis of the depth of burning]. Br J Surg. 1953;40:588-96.

5. Shupp JW, Nasabzadeh TJ, Rosenthal DS, Jordan MH, Fidler P, Jeng JC. A review of the local pathophysiologic bases of burn wound progression. J Burn Care Res. 2010;31:849-73.

6. Heylen L, Thienpont B, Naesens M, Busschaert P, Depreeuw J, Smeets D, et al. Ischemia-Induced DNA Hypermethylation during Kidney Transplant Predicts Chronic Allograft Injury. J. Am. Soc. Nephrol. 2018;29:1566-76.

7. Yuan Y, Zheng Y, Zhang X, Chen Y, Wu X, Wu J, et al. BNIP3L/NIX-mediated mitophagy protects against ischemic brain injury independent of PARK2. Autophagy. 2017;13:1754-66.

8. Tang C, Han H, Yan M, Zhu S, Liu J, Liu Z, et al. PINK1-PRKN/PARK2 pathway of mitophagy is activated to protect against renal ischemia-reperfusion injury. Autophagy. 2018;14:880-97.

9. Ham PB, Raju R. Mitochondrial function in hypoxic ischemic injury and influence of aging. Progress in Neurobiology. 2017;157:92-116.

10. Anzell AR, Maizy R, Przyklenk K, Sanderson TH. Mitochondrial Quality Control and Disease: Insights into Ischemia-Reperfusion Injury. Molecular Neurobiology. Springer US; 2018;55:2547-64.

11. Tan J-Q, Zhang H-H, Lei Z-J, Ren P, Deng C, Li X-Y, et al. The roles of autophagy and apoptosis in burn wound progression in rats. Burns. 2013;39:1551-6.

12. Szczesny B, Brunyánszki A, Ahmad A, Oláh G, Porter C, Toliver-Kinsky T, et al. Time-Dependent and Organ-Specific Changes in Mitochondrial Function, Mitochondrial DNA Integrity, Oxidative Stress and Mononuclear Cell Infiltration in a Mouse Model of Burn Injury. PLoS ONE. 2015;10:e0143730.

13. Liang $W-Y$, Tang L-X, Yang Z-C, Huang YS. Calcium induced the damage of myocardial mitochondrial respiratory function in the early stage after severe burns. Burns. 2002;28:143-6.

14. Padfield KE, Astrakas LG, Zhang Q, Gopalan S, Dai G, Mindrinos MN, et al. Burn injury causes mitochondrial dysfunction in skeletal muscle. Proc Natl Acad Sci USA. 2005;102:5368-73.

15. Wang XM, Chen KM, Shi Y, Shi HP. Functional changes of the NADH respiratory chain in rat-liver mitochondria and the content changes of high-energy phosphate groups in rat liver and heart during the early phase of burn injury. Burns. 1990;16:377-80.

16. Dong YL, Sheng CY, Herndon DN, Waymack JP. Metabolic abnormalities of mitochondrial redox potential in postburn multiple system organ failure. Burns. 1992;18:283-6.

17. Silveira PCL, Venâncio M, Souza PS, Victor EG, de Souza Notoya F, Paganini CS, et al. Iontophoresis with gold nanoparticles improves mitochondrial activity and oxidative stress markers of burn wounds. Mater Sci Eng C Mater Biol Appl. 2014;44:380-5.

18. Kourilsky P. The natural defense system and the normative self model. F1000Res. F1000 Research Limited; 2016;5:797. 
19. Wei $H$, Liu L, Chen Q. Selective removal of mitochondria via mitophagy: distinct pathways for different mitochondrial stresses. Biochim. Biophys. Acta. 2015;1853:2784-90.

20. Shen Z, Zheng Y, Wu J, Chen Y, Wu X, Zhou Y, et al. PARK2-dependent mitophagy induced by acidic postconditioning protects against focal cerebral ischemia and extends the reperfusion window. Autophagy. 2017;13:473-85.

21. Zhang W, Siraj S, Zhang R, Chen Q. Mitophagy receptor FUNDC1 regulates mitochondrial homeostasis and protects the heart from I/R injury. Autophagy. 2017;13:1080-1.

22. $\mathrm{Wu} \mathrm{H}$, Chen Q. Hypoxia activation of mitophagy and its role in disease pathogenesis. Antioxidants \& Redox Signaling. 2015;22:1032-46.

23. Guo SX, Jin YY, Fang Q, You CG, Wang XG, Hu XL, et al. Beneficial effects of hydrogen-rich saline on early burn-wound progression in rats. PLoS ONE. Public Library of Science; 2015;10:e0124897.

24. Eski M, Ozer F, Firat C, Alhan D, Arslan N, Senturk T, et al. Cerium nitrate treatment prevents progressive tissue necrosis in the zone of stasis following burn. Burns. 2012;38:283-9.

25. Guo SX, Zhou H-L, Huang C-L, You CG, Fang Q, Wu P, et al. Astaxanthin attenuates early acute kidney injury following severe burns in rats by ameliorating oxidative stress and mitochondrial-related apoptosis. Marine Drugs. Multidisciplinary Digital Publishing Institute; 2015;13:2105-23.

26. Greenhalgh DG. Management of Burns. N. Engl. J. Med. 2019;380:2349-59.

27. Gravante G, Delogu D, Sconocchia G. "Systemic apoptotic response” after thermal burns. Apoptosis. 2007;12:259-70.

28. Latha B, Babu M. The involvement of free radicals in burn injury: a review. Burns. 2001;27:309-17.

29. Arturson G. Pathophysiology of the burn wound and pharmacological treatment. The Rudi Hermans Lecture, 1995. Burns. 1996. pp. 255-74.

30. Colpaert K, Hoste EA. Acute kidney injury in burns: a story of volume and inflammation. Crit Care. 2008;12:192.

31. Costantini TW, Peterson CY, Kroll L, Loomis WH, Putnam JG, Wolf P, et al. Burns, inflammation, and intestinal injury: protective effects of an anti-inflammatory resuscitation strategy. J Trauma. 2009;67:1162-8.

32. Huang Y, Li Z, Yang Z. Roles of ischemia and hypoxia and the molecular pathogenesis of post-burn cardiac shock. Burns. 2003;29:828-33.

33. Palmieri T, Lavrentieva A, Greenhalgh DG. Acute kidney injury in critically ill burn patients. Risk factors, progression and impact on mortality. Elsevier Ltd and International Society of Burns Injuries; 2010;36:205-11.

34. Hoeksema H, Van de Sijpe K, Tondu T, Hamdi M, Van Landuyt K, Blondeel P, et al. Accuracy of early burn depth assessment by laser Doppler imaging on different days post burn. Burns. 2009;35:36-45.

35. Prindeze NJ, Hoffman HA, Ardanuy JG, Zhang J, Carney BC, Moffatt LT, et al. Active Dynamic Thermography is a Sensitive Method for Distinguishing Burn Wound Conversion. J Burn Care Res. 2016;37:e559-68. 
36. McElroy GS, Chandel NS. Mitochondria control acute and chronic responses to hypoxia. Experimental Cell Research. 2017;356:217-22.

37. Guo SX, Fang Q, You CG, Jin YY, Wang XG, Hu XL, et al. Effects of hydrogen-rich saline on early acute kidney injury in severely burned rats by suppressing oxidative stress induced apoptosis and inflammation. J Transl Med. 2015;13:183.

38. Fang Q, Guo S, Zhou H, Han R, Wu P, Han C. Astaxanthin protects against early burn-wound progression in rats by attenuating oxidative stress-induced inflammation and mitochondria-related apoptosis. Sci Rep. Nature Publishing Group; 2017;7:41440-13.

39. Mizumura K, Choi AMK, Ryter SW. Emerging role of selective autophagy in human diseases. Front Pharmacol. 2014;5:244.

40. Tang C, Han H, Liu Z, Liu Y, Yin L, Cai J, et al. Activation of BNIP3-mediated mitophagy protects against renal ischemia-reperfusion injury. Cell Death and Disease. Nature Publishing Group; 2019;10:677-15.

41. Livingston MJ, Wang J, Zhou J, Wu G, Ganley IG, Hill JA, et al. Clearance of damaged mitochondria via mitophagy is important to the protective effect of ischemic preconditioning in kidneys. Autophagy. 2019;15:2142-62.

42. Wu H, Wang Y, Li W, Chen H, Du L, Liu D, et al. Deficiency of mitophagy receptor FUNDC1 impairs mitochondrial quality and aggravates dietary-induced obesity and metabolic syndrome. Autophagy. 2019;15:1882-98.

43. Zheng $Y$, Wu $X$, Chen $Z$, Zhang $X$. Come and eat: mitochondrial transport guides mitophagy in ischemic neuronal axons. Autophagy. 2019;15:1483-4.

44. He Q, Li Z, Meng C, Wu J, Zhao Y, Zhao J. Parkin-Dependent Mitophagy is Required for the Inhibition of ATF4 on NLRP3 Inflammasome Activation in Cerebral Ischemia-Reperfusion Injury in Rats. Cells. $2019 ; 8$.

45. Chourasia AH, Boland ML, Macleod KF. Mitophagy and cancer. Cancer Metab. BioMed Central; 2015;3:4-11.

46. Chourasia AH, Macleod KF. Tumor suppressor functions of BNIP3 and mitophagy. Autophagy. 2015;11:1937-8.

47. Li L, Tan J, Miao Y, Lei P, Zhang Q. ROS and Autophagy: Interactions and Molecular Regulatory Mechanisms. Cell Mol Neurobiol. Springer US; 2015;35:615-21.

48. Wang GL, Jiang BH, Rue EA, Semenza GL. Hypoxia-inducible factor 1 is a basic-helix-loop-helix-PAS heterodimer regulated by cellular 02 tension. Proc Natl Acad Sci USA. 1995;92:5510-4.

49. Ivan M, Kondo K, Yang H, Kim W, Valiando J, Ohh M, et al. HIFalpha targeted for VHL-mediated destruction by proline hydroxylation: implications for $\mathrm{O} 2$ sensing. Science. American Association for the Advancement of Science; 2001;292:464-8.

50. Semenza GL, Nejfelt MK, Chi SM, Antonarakis SE. Hypoxia-inducible nuclear factors bind to an enhancer element located 3 ' to the human erythropoietin gene. Proc Natl Acad Sci USA. 1991;88:5680-4. 
51. Maxwell PH, Wiesener MS, Chang GW, Clifford SC, Vaux EC, Cockman ME, et al. The tumour suppressor protein VHL targets hypoxia-inducible factors for oxygen-dependent proteolysis. Nature. Nature Publishing Group; 1999;399:271-5.

52. Jaakkola P, Mole DR, Tian YM, Wilson MI, Gielbert J, Gaskell SJ, et al. Targeting of HIF-alpha to the von Hippel-Lindau ubiquitylation complex by 02 -regulated prolyl hydroxylation. Science. American Association for the Advancement of Science; 2001;292:468-72.

53. Kumar H, Choi D-K. Hypoxia Inducible Factor Pathway and Physiological Adaptation: A Cell Survival Pathway? Mediators of Inflammation. Hindawi; 2015;2015:584758-11.

54. Lee J-W, Bae S-H, Jeong J-W, Kim S-H, Kim K-W. Hypoxia-inducible factor (HIF-1)alpha: its protein stability and biological functions. Exp. Mol. Med. 2004;36:1-12.

55. Xing D, Liu L, Marti GP, Zhang X, Reinblatt M, Milner SM, et al. Hypoxia and hypoxia-inducible factor in the burn wound. Wound Rep Reg. 2011;19:205-13.

56. Zhang T, Xue L, Li L, Tang C, Wan Z, Wang R, et al. BNIP3 Protein Suppresses PINK1 Kinase Proteolytic Cleavage to Promote Mitophagy. Journal of Biological Chemistry. 2016;291:21616-29.

57. Stieg DC, Cooper KF. Parkin New Cargos: a New ROS Independent Role for Parkin in Regulating Cell Division. React Oxyg Species (Apex). 2016;2:315-24.

\section{Figures}




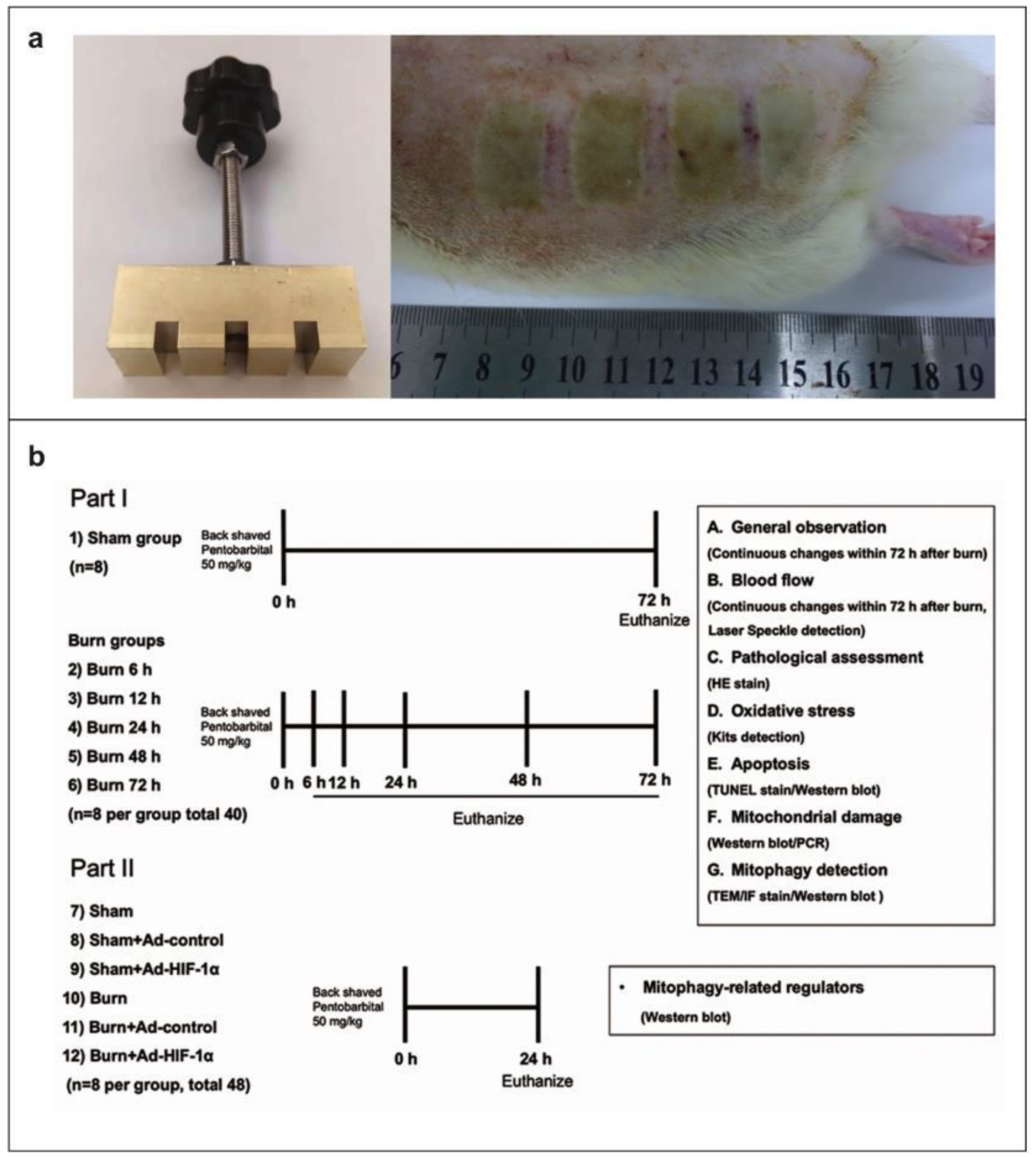

\section{Figure 1}

Animal model and Study design. a The customized rectangular brass comb (Left) and a representative photography of "comb" scald model (Right); b Schematic diagram of experimental design and animal grouping. 


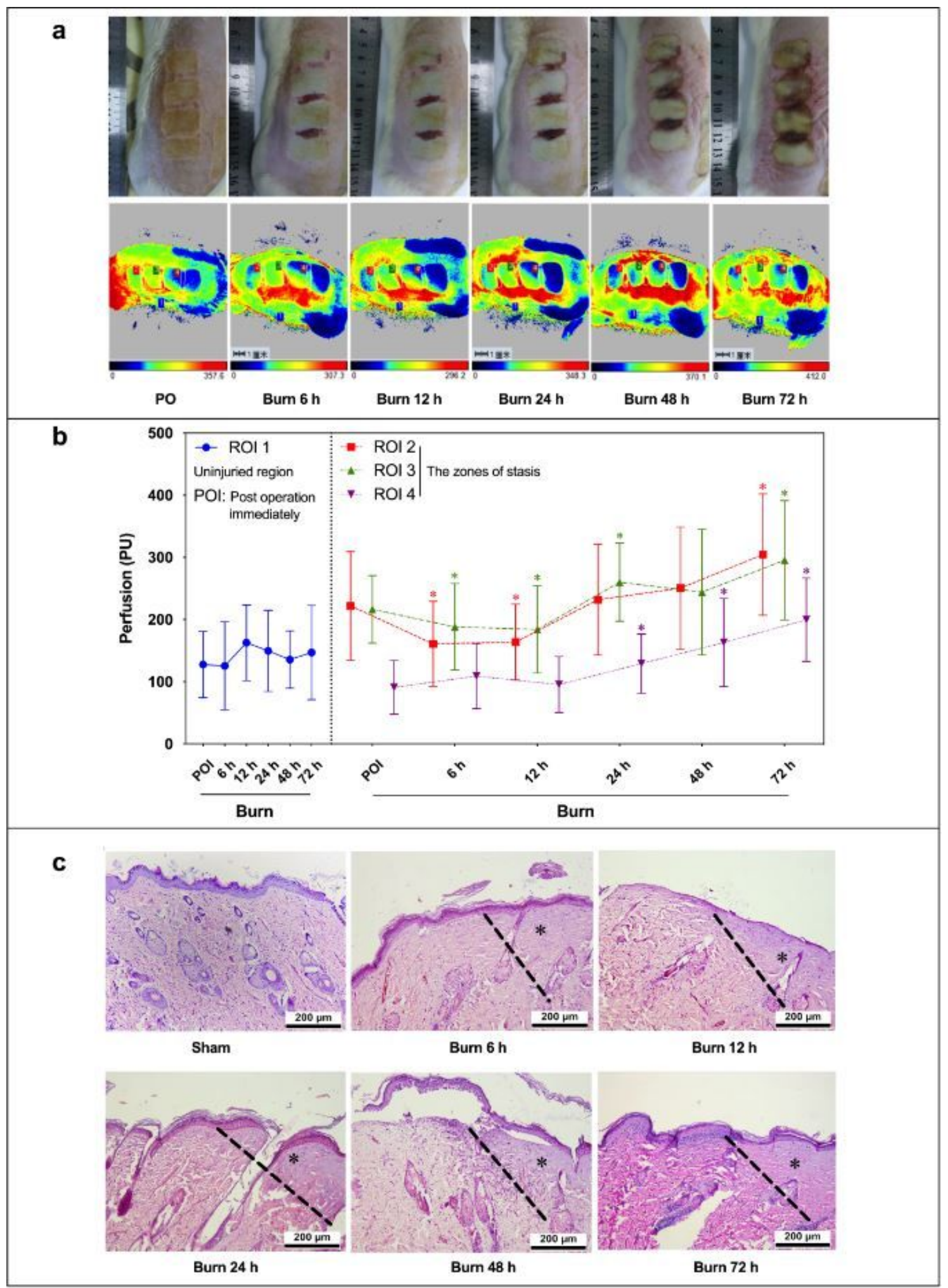

\section{Figure 2}

Blood-flow and pathologic changes in the zone of stasis with time after burn injury. a General observation (Upper) and Laser Dopler detection of blood-flow in burn-wounds(Lower); b Assessment on the local perfusion of uninjuried region ( $\mathrm{ROI} 1$ ) and the zones of stasis (ROI 2, 3, 4) after burn; c Representative HEstained slices present the special histological changes in the different time groups. A dotted line marks 
the progression boundary, while the initial burn regions are labelled with asterisks, presenting obvious hyalinization. ${ }^{*} p<0.05$ versus the POI group. Scale bars $=200 \mu \mathrm{m}, \mathrm{n}=8$ per group.

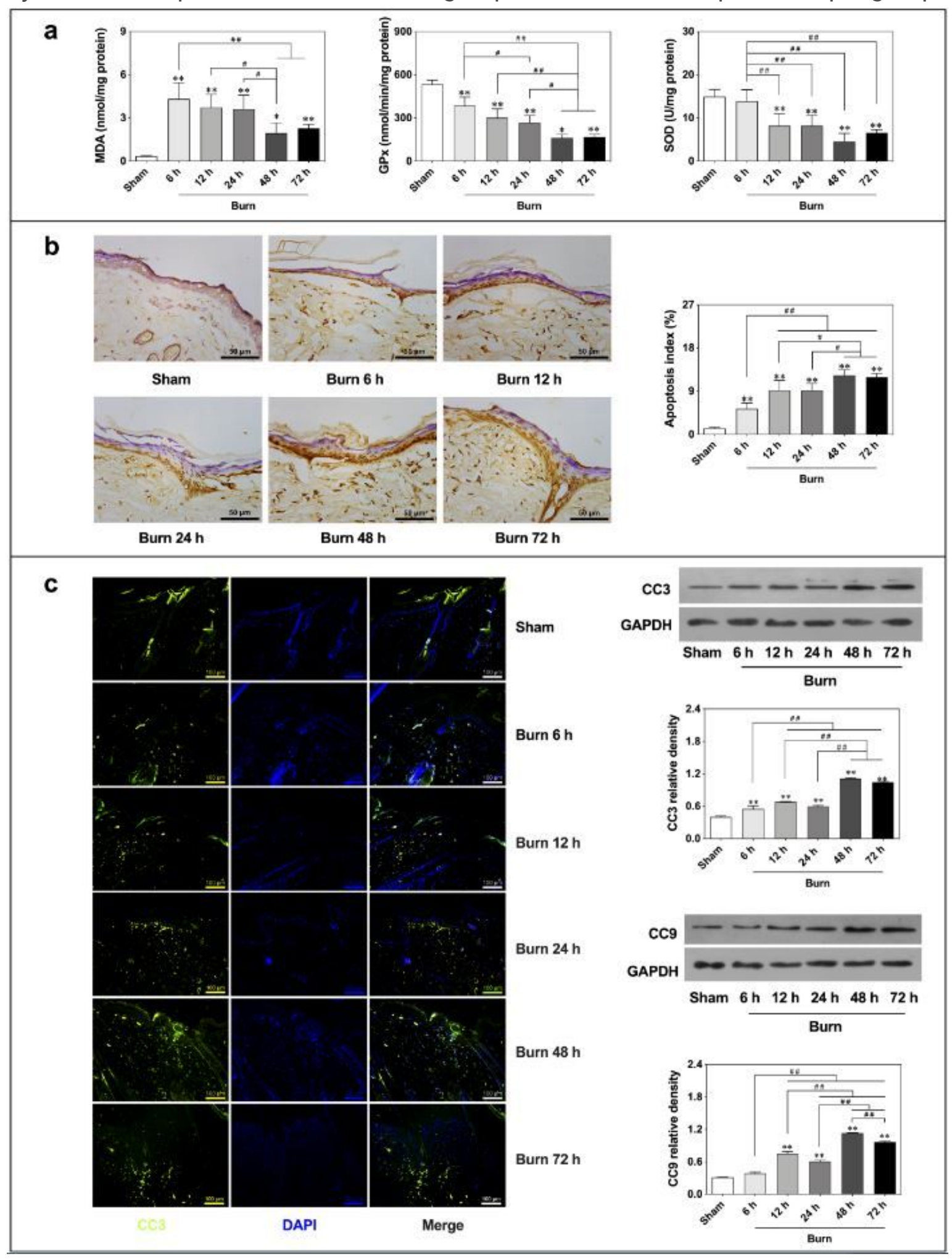

\section{Figure 3}

Assessment of oxidative stress and apoptosis in burn-wounds. a Time-related changes of indicators of oxidative stress; b Apoptosis index assessment based on TUNEL staining; c Immunofluorescence staining shows the distribution of CC3, and western blot detects the expression of CC9 and CC3. Scale 
bars $=100 \mu \mathrm{m}$. The results are expressed as the means \pm SDs. ${ }^{*} p<0.05$ and ${ }^{* *} p<0.01$ versus the sham group; $\# p<0.05, \# \# p<0.01$, and $n s p>0.05$. $n=6$ per group. CC3, cleaved caspase 3 ; CC9, cleaved caspase 9.

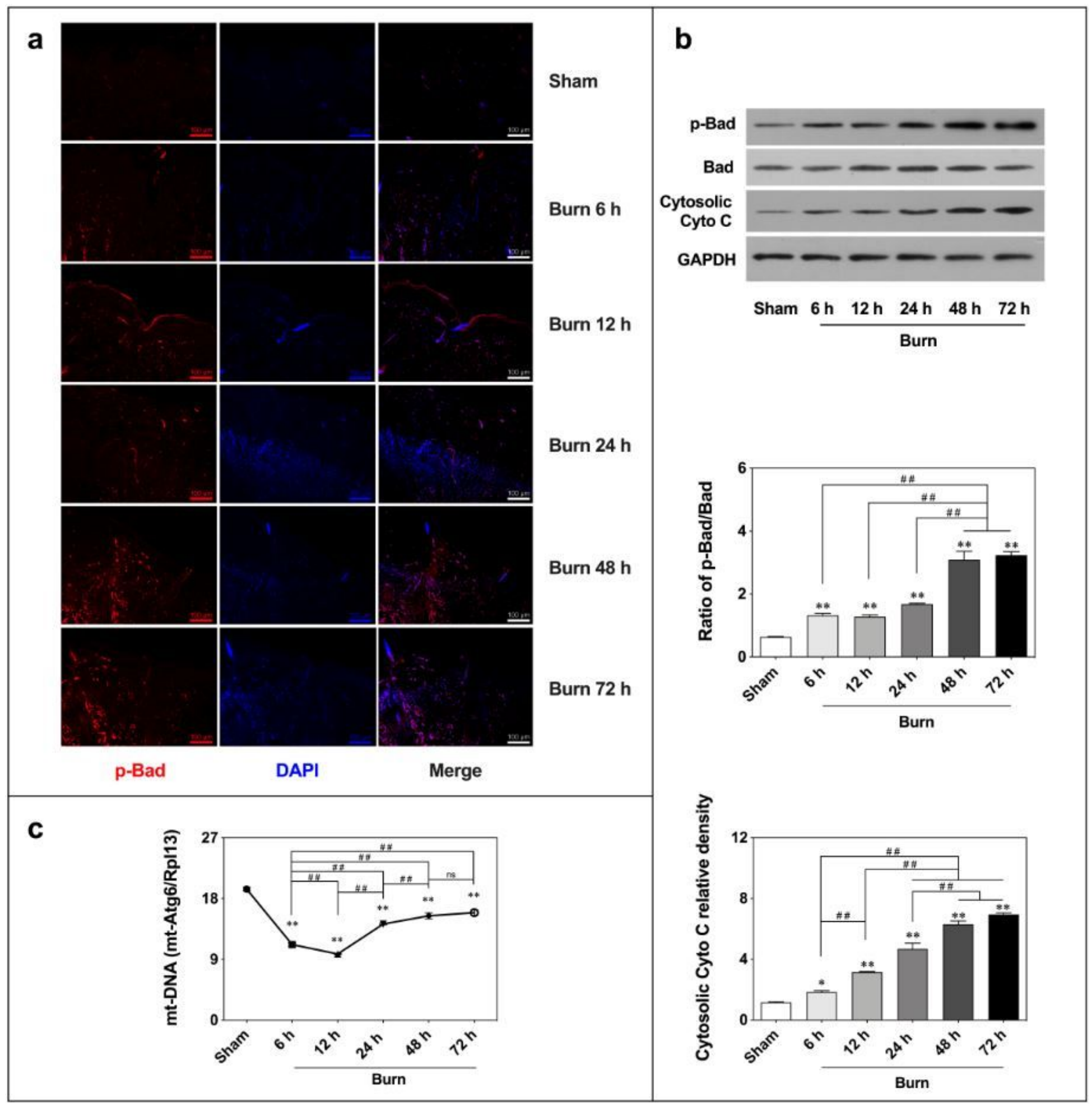

\section{Figure 4}

Impaired mitochondria cause activation of mitochondria-related apoptotic pathway. a Immunofluorescence staining of $\mathrm{p}$-Bad; $b$ Western blot analysis of $\mathrm{p}$-Bad/Bad and cytosolic Cytochrome $\mathrm{C}$ (Cyto C); c The time change of relative mitochondrial DNA (mtDNA) level is detected by real-time PCR, 
which is presented as the mt-Atp6 (mitochondria-encoded DNA) versus Rpl13 (nucleus-encoded DNA) ratio in cells of the zone of stasis. Scale bars $=100 \mu \mathrm{m}$. The results are expressed as the means \pm SDs. *p $<0.05$ and ${ }^{* *} \mathrm{p}<0.01$ versus the sham group; $\# \mathrm{p}<0.05$, \#\#p<0.01, and $\mathrm{nsp}>0.05$. $\mathrm{n}=6$ per group.

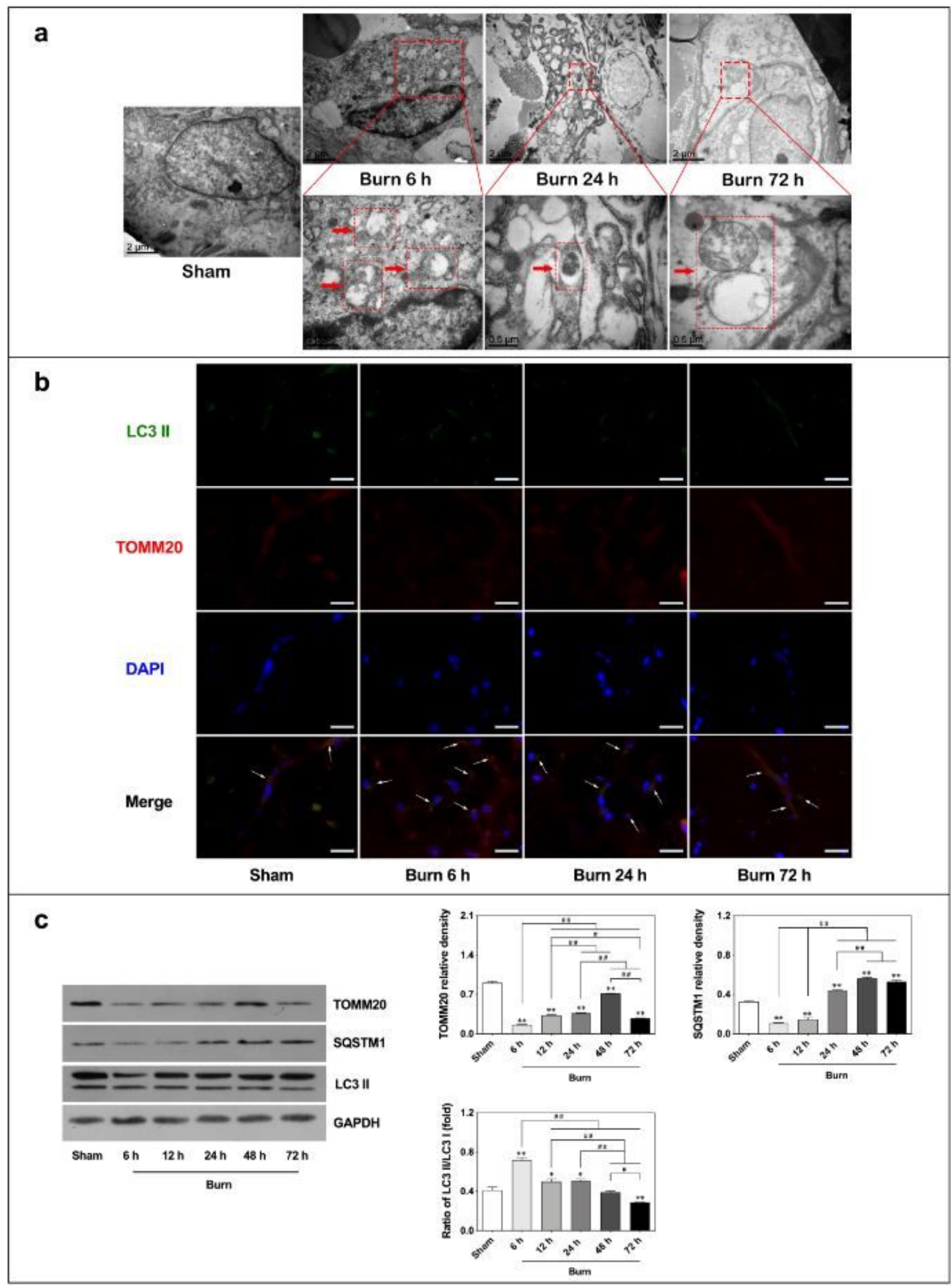

Figure 5

Detection of mitophagy in the zone of stasis post-burn. a Ultrastructural investigation of mitophagy via Transmission Electron Microscope; b Immunofluorescence dual staining of LC3 II and TOMM20 for 
marking the location of mitophagy in the selected time-windows; c Western blot results of TOMM20, SQSTM1/P62, LC3 II. Scale bars $=2$ or $1,0.5 \mu \mathrm{m}$ for TEM. Scale bars $=100 \mu \mathrm{m}$ for immunofluorescence staining. The results are expressed as the means \pm SDs. ${ }^{*} p<0.05$ and ${ }^{* \star} p<0.01$ versus the sham group; $\# p<0.05, \# \# p<0.01$, and $n s p>0.05 . n=6$ per group.

a

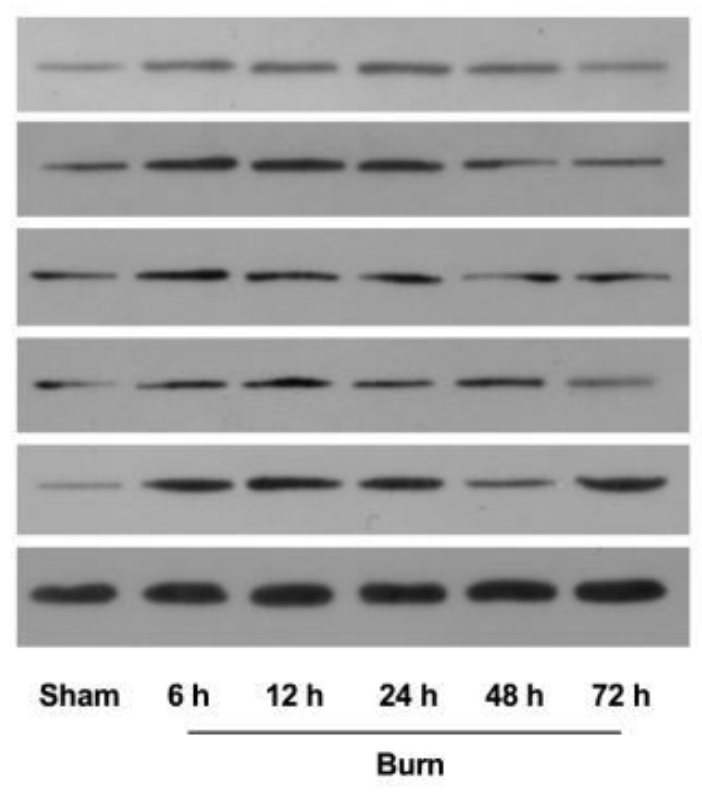

PARKIN

PINK1

BNIP3

BNIP3L

FUNDC1

GAPDH
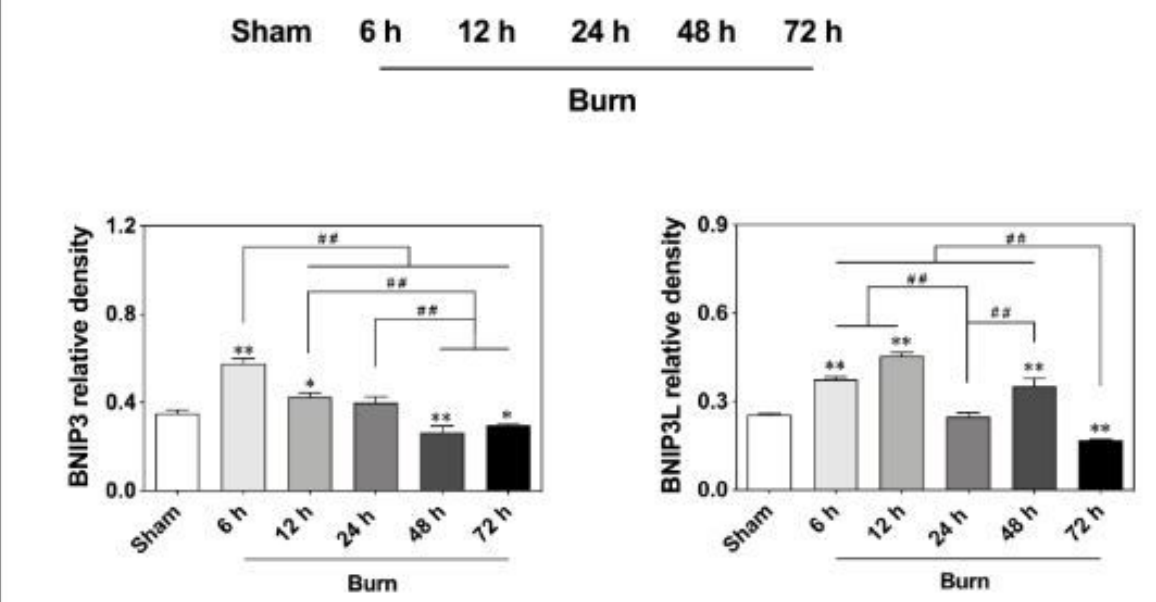
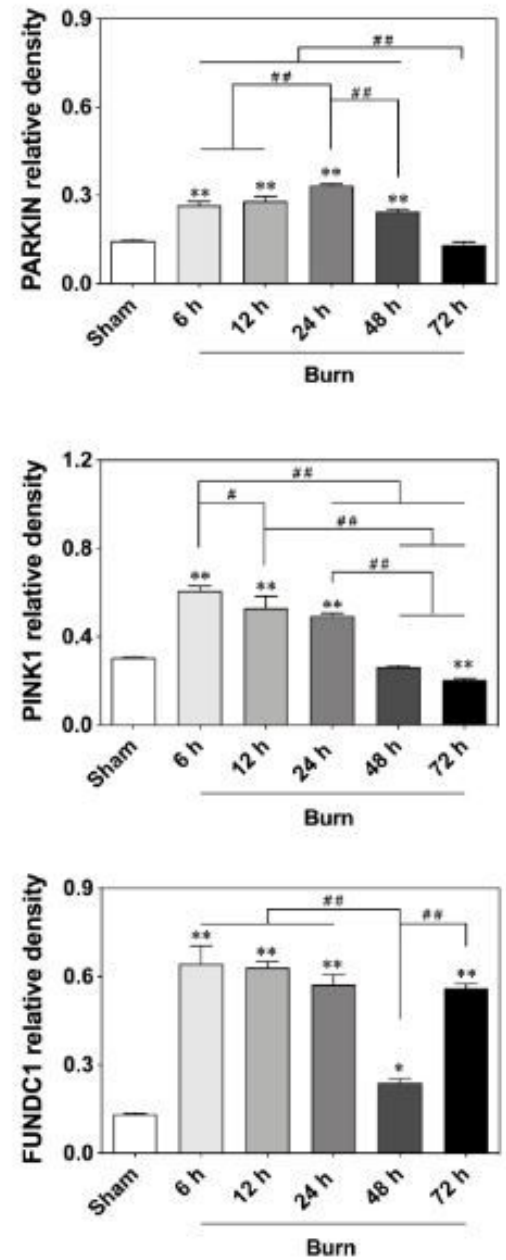

b

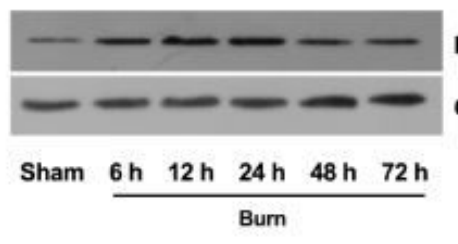

HIF- $1 \alpha$

GAPDH

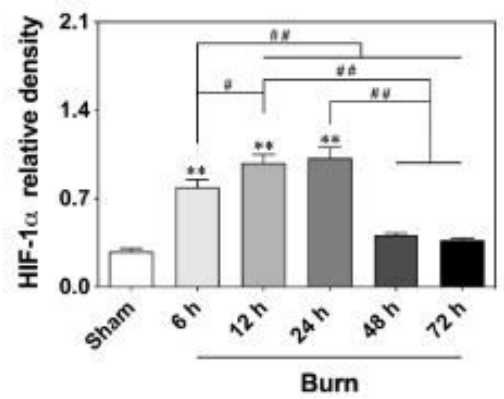

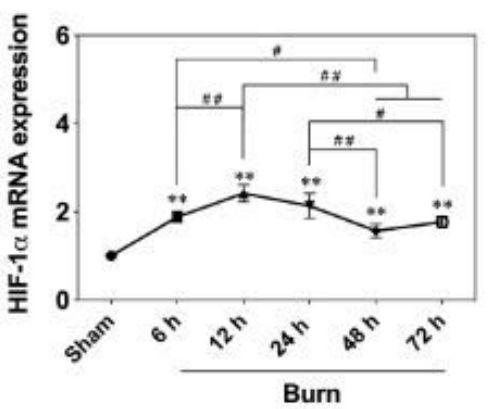

Figure 6 
Potential regulators involved in mitophagy of burn-wounds. a Protein expression of mitophagy regulators based on western blot. b Western blot and real-time PCR detection of HIF-1区. The results are expressed as the means \pm SDs. ${ }^{*} p<0.05$ and ${ }^{* *} p<0.01$ versus the sham group; $\# p<0.05$, \#\#p $<0.01$, and $n s p>0.05$. $\mathrm{n}=6$ per group.

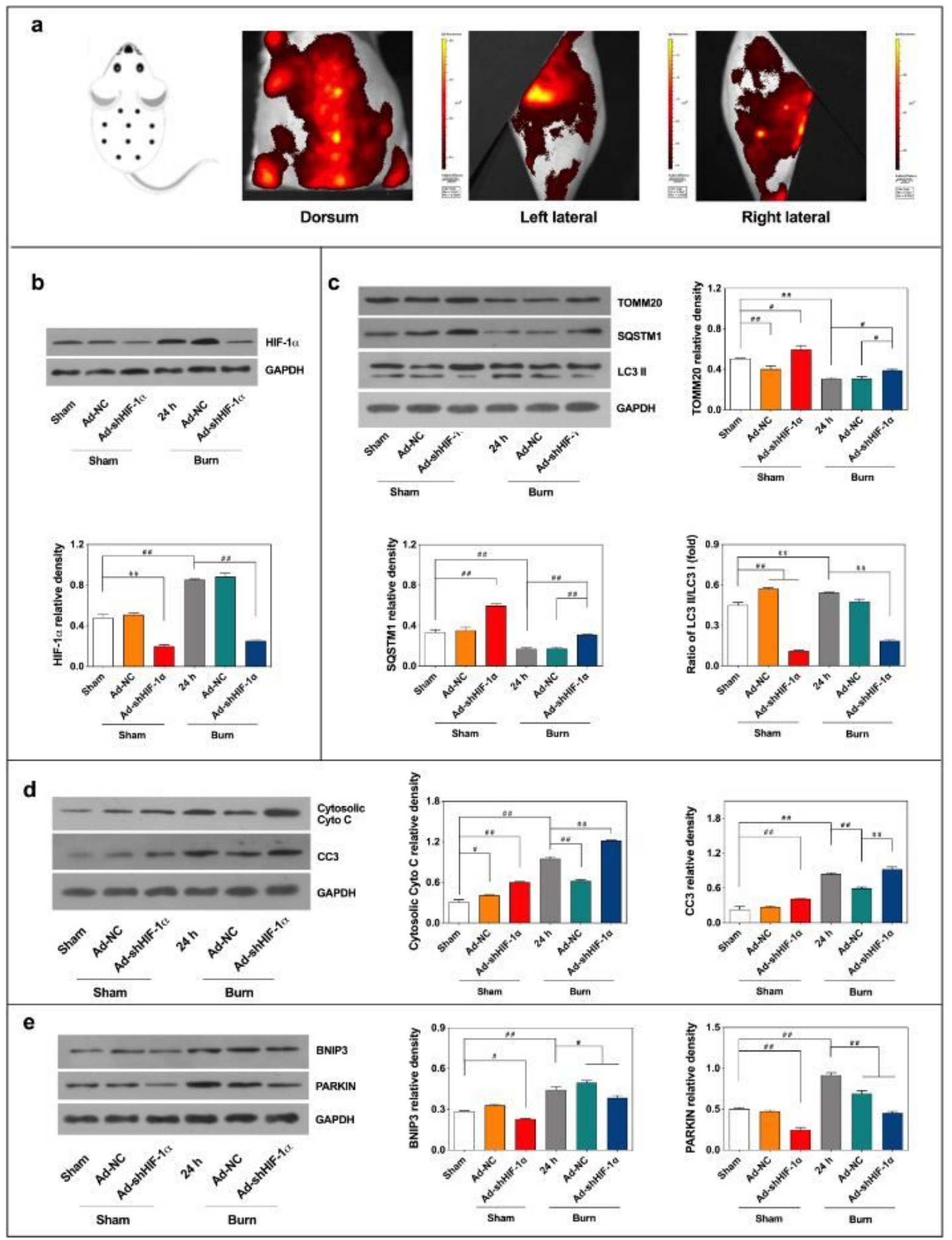

Figure 7 
Adenovirus-based HIF-1】 silence causes an inhibition of mitophagy and increase of apoptosis in the zone of stasis, and both BNIP3 and PARKIN works downstream of HIF-1区. a Biological in vivo imaging 3 days after adenovirus application; $b$ The expression changes of HIF-1囚 after adenovirus application; $c$ The changes of mitophagy indicators by western blot under HIF-1Q silence; $d$ The changes of activation of mitochondrial apoptotic pathway after HIF-1区 silence; e Western blot results of BNIP3 and PARKIN in the zone of stasis following shHIF-1区 introduction. The results are expressed as the means $\pm S D s$. $\# p<0.05$, $\# \# p<0.01$, and $n s p>0.05 . n=6$ per group.

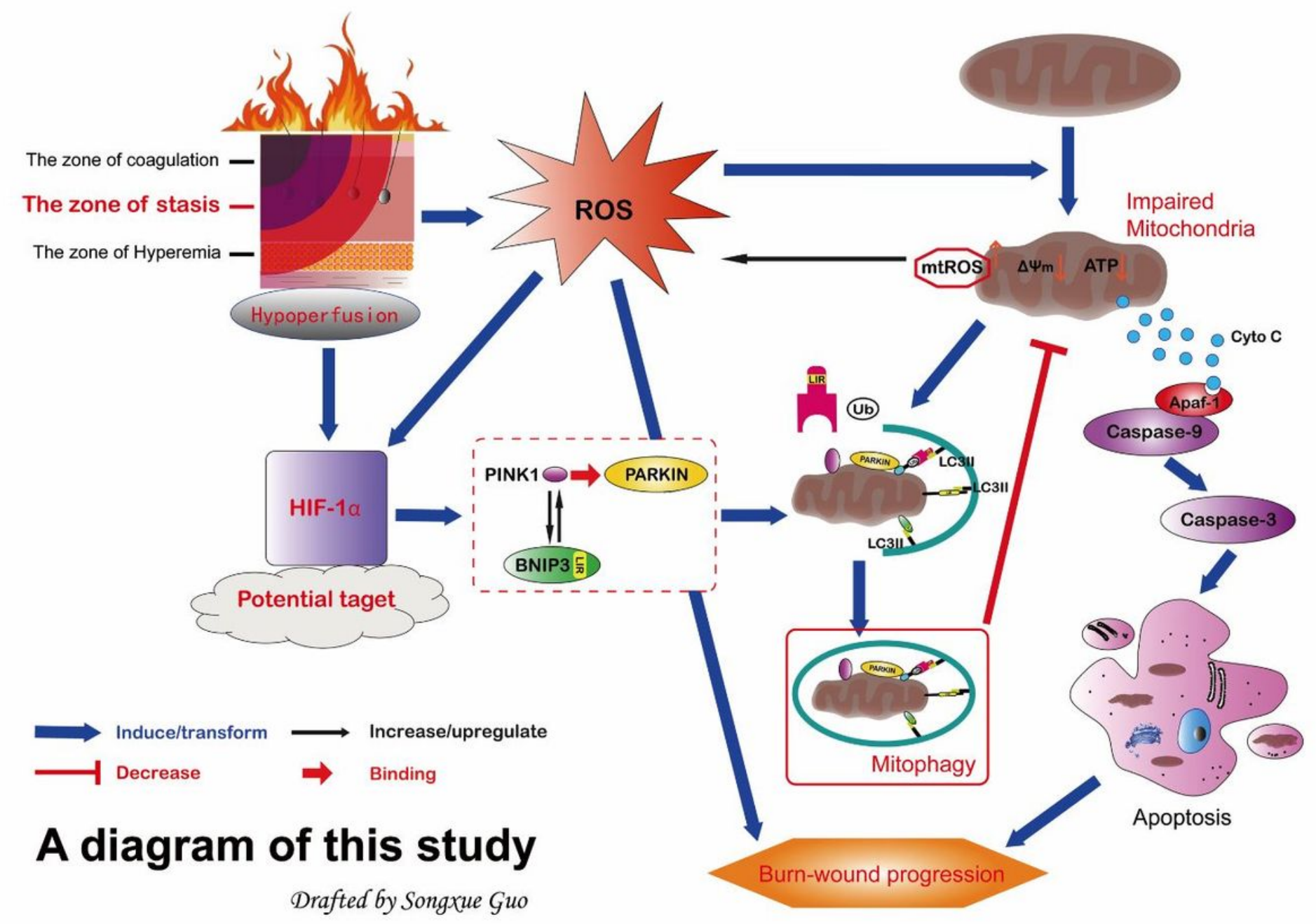

\section{Figure 8}

Schematic diagram of the role of mitophagy in burn-wound progression. 